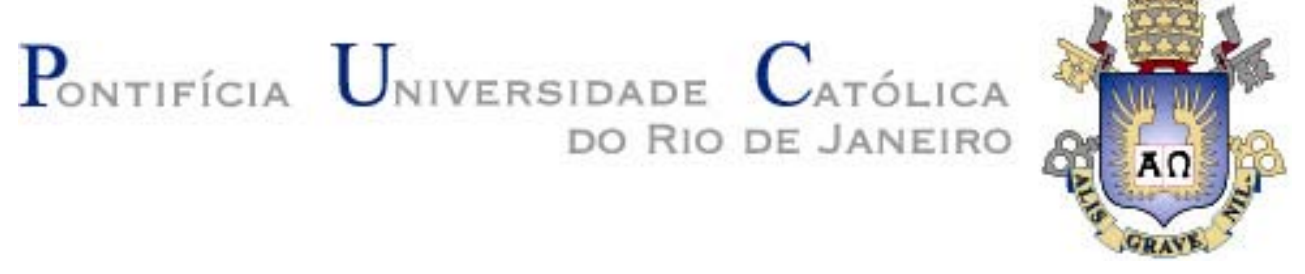

Samuel de Oliveira Cardoso

Análise de Investimento de Capital na Indústria Brasileira de papel e celulose por meio da Teoria das Opções Reais: O Caso da Fibria Celulose S.A.

Dissertação de Mestrado

Dissertação apresentada como requisito parcial para obtenção do título de Mestre pelo Programa de PósGraduação em Engenharia Industrial da PUC-Rio.

Orientador: Prof. Carlos Patrício Samanez 
Samuel de Oliveira Cardoso

\title{
Análise de Investimento de Capital na Indústria Brasileira de papel e celulose por meio da Teoria das Opções Reais: O Caso da Fibria Celulose S.A.
}

\begin{abstract}
Dissertação apresentada como requisito parcial para obtenção do titulo de Mestre (opção profissional) pelo Programa de Pós-Graduação em Engenharia de Produção da PUC-Rio. Aprovada pela Comissão Examinadora abaixo assinada.
\end{abstract}

Prof. Carlos Patrício Samanez Orientador e Presidente Departamento de Engenharia Industrial - PUC-Rio

Prof. Marco Antonio Guimarães Dias Departamento de Engenharia Industrial - PUC-Rio

Prof. André Barreira da Silva Rocha Departamento de Engenharia Industrial - PUC-Rio

Prof. José Eugenio Leal Coordenador Setorial do Centro Técnico Científico - PUC-Rio Rio de Janeiro, 03 de Novembro de 2014 
Todos os direitos reservados. É proibida a reprodução total ou parcial do trabalho sem autorização do autor, do orientador e da universidade.

\section{Samuel de Oliveira Cardoso}

Graduou-se cum laude em Licenciatura em Matemática pela Universidade Federal do Rio de Janeiro em Dezembro de 2006. Graduou-se em Administração Pública e de Empresas pela Universidade Federal Rural do Rio de Janeiro em Setembro de 2007. Pós-graduou-se em MBE em Economia e Gestão da Sustentabilidade pela Universidade Federal do Rio de Janeiro em Maio de 2010. No período de 2001 a 2007, trabalhou, no Comando da Aeronáutica, em cargo de nível técnico na área de Logística Militar. Entre 2007 e 2008, foi Professor I de Matemática, do Quadro Permanente do Município do Rio de Janeiro. De 2008 a 2009, foi Professor Substituto do Departamento de Matemática (DEMAT) da Universidade Federal Rural do Rio de Janeiro. Desde 2008, trabalha como Administrador do Banco Nacional de Desenvolvimento Econômico e Social (BNDES) na Área de Planejamento.

Ficha Catalográfica

Cardoso, Samuel de Oliveira

Análise de investimento de capital na indústria brasileira de papel e celulose por meio da teoria das opções reais: o caso da Fibria Celulose S.A. / Samuel de Oliveira Cardoso ; orientador: Carlos Patrício Samanez. - 2014.

148 f. : il. (color.) ; $30 \mathrm{~cm}$

Dissertação (mestrado)-Pontifícia Universidade Católica do Rio de Janeiro, Departamento de Engenharia Industrial, 2014.

Inclui bibliografia

1. Engenharia Industrial - Teses. 2. Celulose e papel. 3. Opções reais. 4. Processamento estocástico. 5. Reversão à média. 6. Simulação de Monte Carlo. 7. Método binomial. I. Samanez, Carlos Patrício. II. Pontifícia Universidade Católica do Rio de Janeiro. Departamento de Engenharia Industrial. III. Título. 


\section{Agradecimentos}

Ao meu avô, João Norberto (in memoriam), pelo exemplo que me deixou nos nossos anos de convivência; aos meus pais, pelo carinho, dedicação e ensinamentos deixados: minha mãezinha, professora Moema e meu pai Rivaldo.

À minha querida e amada esposa, Aline, sempre presente em momentos importantes da minha vida. Ao meu orientador Carlos PatricioSamanez e aos demais professores da Comissão Examinadora pelos conhecimentos transmitidos.

Aos amigos Aquiles Poletti, Administrador do BNDES; e, Roberto Hollmann, Engenheiro da Petrobras e colega de Mestrado, pela generosidade na troca de conhecimentos.

À Secretária do Mestrado, Érica da Hora, pela sua dedicação aos assuntos acadêmicos.

Por fim, ao BNDES, pelo financiamento integral a este Curso de Mestrado. 


\section{Resumo}

Cardoso, Samuel de Oliveira; Samanez, Carlos Patrício. Análise de Investimento de Capital na Indústria Brasileira de papel e celulose por meio da Teoria das Opções Reais: O Caso da Fibria Celulose S. A. Rio de Janeiro, 2014. 148p. Dissertação de Mestrado - Departamento de Engenharia Industrial, Pontifícia Universidade Católica do Rio de Janeiro.

O presente trabalho tem como objetivo final a verificação da aplicabilidade da Teoria das Opções Reais (TOR) em investimentos de papel e celulose, considerando o Movimento de Reversão à Média (MRM) nos fatores de risco, dado um modelo de gerenciamento de curto prazo, no âmbito de um estudo de caso da Fibria Celulose S.A. para o setor de papel e celulose no Brasil. Nesta dissertação, testa-se a aderência da série histórica de preços da celulose de fibra curta da Fibria, no período entre 2003 e 2013, a um modelo estocástico de reversão à média, sendo este modelo validado para o presente estudo. Uma vez o modelo validado, determinam-se os parâmetros para realização de cálculos e análises fundamentais para se chegar aos objetivos intermediários, etapa preliminar aos resultados do objetivo final. Dentre os cálculos e análises citados, ressaltam-se: determinação dos VPLs dinâmicos e os valores das Opções Reais europeias sequenciais para a Simulação de Monte Carlo com Processo Neutro ao Risco; construção e análise da Árvore Binomial com Processo Neutro ao Risco; construção e análise das Regiões de Gatilho para preços e lucros marginais em um Processo Real; comparação das Regiões de Gatilho com as determinadas pelas Árvores Binomiais. Assim, com tais análises, confirma-se, nesta dissertação, a aplicabilidade da Teoria das Opções Reais na Análise de Investimento no setor celulósico-papeleiro.

\section{Palavras-chave}

Celulose e papel; opções reais; processos estocásticos; reversão à média; simulação de Monte Carlo; método binomial. 


\section{Abstract}

Cardoso, Samuel de Oliveira; Samanez, Carlos Patrício (Advisor). Analysis of Capital Investment in the Brazilian pulp and paper industry by means of the Real Options Theory: The case of Fibria Celulose S. A. Rio de Janeiro, 2014. 148p. MSc. Dissertation - Departamento de Engenharia Industrial, Pontifícia Universidade Católica do Rio de Janeiro.

The present work has the ultimate purpose of verifying the applicability of the Real Options Theory in the pulp and paper investment, considering the Mean Reversion Movement in the risk factors, given a short-term management model, within a study of Fibria Celulose S.A. for the pulp and paper industry in Brazil. This dissertation tests the adherence of Fibria's short fiber pulp historical price series, between 2003 and 2013 to a stochastic mean reversion model, being this model validated in the present study. Once the model is validated, the parameters for calculations and fundamental analyzes are determined to reach intermediate goals, preliminary step to the results of the final goal. Among the cited calculations and analyzes, it is emphasized: determination of dynamic NPVs and values of the sequential European Real Options for the Monte Carlo Simulation with Risk Neutral Process; construction and analysis of the Binomial Tree with Risk Neutral Process; construction and analysis of the Trigger Regions for prices and marginal profits in a Real Case; Comparison between Trigger Regions and those determined by the Binomial Trees.So with such analyzes, it is confirmed, in this work, the applicability of the Real Option Theory on Investment Analysis in pulp and paper industry.

\section{Keywords}

Pulp and Paper; Real Options; Stochastic Process; Mean Reversion; Monte Carlo Simulation; Binomial Method. 


\section{Sumário}

1. Introdução 13

1.1. Posicionamento Acadêmico da Dissertação 15

1.2. Estrutura da Dissertação 15

$\begin{array}{ll}\text { 1.3. Relevância do Estudo } & 17\end{array}$

1.4. Aspectos Metodológicos 18

1.5. Definição do Problema e dos Objetivos 18

$\begin{array}{ll}\text { 1.5.1. Problema Investigado } & 19\end{array}$

1.5.2. Objetivos Intermediários 19

1.5.3. Objetivo Final 20

2. Cadeia Produtiva e Outros Aspectos Setoriais do Setor de Celulose e Papel 21

2.1. Cadeia Produtiva de Papel e Celulose 21

2.1.1. Etapa Florestal 23

2.1.2. Etapa Industrial $\quad 25$

2.2. Outros Aspectos Setoriais 28

2.2.1. Fibria Celulose S/A 30

3. Referencial Teórico 33

3.1. Teoria das Opções Reais 33

3.1.1. Tipos de Opções 35

3.2. Processos Estocásticos $\quad 39$

3.2.1. Processo de Markov 41

3.2.2. Processo de Wiener 41

3.2.3. Processo de Itô 43

3.2.4. Movimento Geométrico Browniano (MGB) 43

3.2.5. Movimento de Reversão à Média (MRM) 46

3.2.6. Validação dos Processos Estocásticos 51

3.2.6.1. Teste de Raiz unitária (Dickey-Fuller) 52

3.2.6.2. Teste da Razão de Variância 55

3.2.7. Modelos de Precificação das Opções 56

3.2.7.1. Modelo de Black e Scholes 56

3.2.7.2. Modelo Binomial $\quad 59$

3.2.7.3. Simulação de Monte Carlo (SMC) 63

4. Metodologia, Aplicações e Resultados 68

4.1. Opções Reais e Fibria S/A $\quad 70$

4.2. A Fibria $\quad 71$

4.3. Premissas $\quad 71$

4.4. Levantamento de Dados 73 
4.5. Escolha e Validação do Processo Estocástico 74

4.5.1. Teste de Dickey-Fuller $\quad 75$

4.5.2. Teste de Razão da Variância 79

4.6. Modelo de Reversão à Média (MRM) 80

4.7. SMC - Processo Neutro ao Risco (MRM) 87

4.8. SMC - Processo Real x Árvore Binomial com Processo

Neutro ao Risco (MRM) 91

4.8.1. SMC - Processo Real $\quad 92$

4.8.2. SMC - Árvore Binomial Neutra ao Risco (MRM) 96

4.8.3. SMC - Comparação das Regiões de Gatilho com os

Valores da Árvore Binomial 101

4.9. Aspectos Gerais e Resultados do Estudo de Caso 102

5. Conclusão 106

5.1. Considerações Finais Sobre o Estudo de Caso 106

5.2. Sugestões para Trabalhos Futuros 108

$\begin{array}{ll}\text { 6. Referências Bibliográficas } & 109\end{array}$

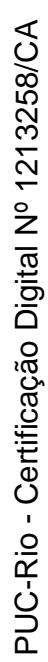

$\begin{array}{ll}\text { 7. Anexos } & 114\end{array}$

7.1. Algumas Definições Adicionais na Etapa Industrial do Processo da Celulose

7.2. Dados Utilizados 115

7.3. Simulações no @Risk 118

7.3.1. Introdução ao @Risk - Add-In do Microsoft Excel para

$\begin{array}{ll}\text { Simulação e Análise de Riscos } & 118 \\ 7.32 \text { Simulações no @Risk - Processo Neutro ao Risco } & 121\end{array}$

7.3.2. Simulações no @Risk - Processo Neutro ao Risco 121

7.3.3. Simulações no @Risk - Processo Real 128 


\section{Lista de tabelas}

Tabela 1 - Etapas do processo fabril da celulose 28

Tabela 2 - Tabela para Árvore Binomial Recombinante $(n=3) \quad 60$

Tabela 3 - Dados gerais $\quad 72$

Tabela 4 - Faixas de preço para o Gatilho 93

Tabela 5 - Faixas de lucro para o Gatilho 95

Tabela 6 - Parâmetros para Árvore Binomial 96

Tabela 7 - Caso geral - Tabela Binomial para $n=12 \quad 97$

Tabela 8 - Análise Binomial Incremental $(n=12)$ com $P_{0}=479,9199$

Tabela 9 - Análise das faixas de preços pelo Método Binomial 98 


\section{Lista de figuras}

Figura 1 - Evolução das produções brasileiras de celulose e papel 28

Figura 2 - Evolução da produção mensal de celulose 30

Figura 3 - Valor da Flexibilidade: Incerteza e Capacidade de

Reagir

Figura 4 - Classificação dos Tipos de Opções Reais 36

Figura 5 - Métodos de Resolução de OR 38

Figura 6 - Gráfico Intuitivo sobre o MGB 44

Figura 7 - Gráfico Intuitivo sobre o MRM 47

Figura 8 - Quadro resumo para estimação de parâmetros do MRM 51

Figura 9 - Árvore Binomial Recombinante $(n=3) \quad 60$

Figura 10 - Inputs, Outputs e Distribuições Probabilísticas 63

Figura 11 - Inputs e Distribuições Probabilísticas no @Risk 64

Figura 12 - Células para os Outputs no @Risk 65

Figura 13 - Outputs e Distribuições Probabilísticas no @Risk 65

Figura 14 - Esquematização das etapas da SMC 67

Figura 15 - Modelo Conceitual para as simulações 70

Figura 16 - Resumo da escolha do processo estocástico 75

Figura 17 - Tela de entrada de Parâmetro do Teste 78

Figura 18 - Teste ADF no EViews 8

Figura 19 - Análise de Regressão no EViews 8 


\section{Lista de gráficos}

Gráfico 1 - Preços deflacionados da celulose de fibra curta 73

Gráfico 2 - Histograma e Estatísticas 74

Gráfico 3 - Ln dos preços deflacionados da celulose de fibra curta 76

Gráfico 4 - Histograma e Estatísticas (Ln) 77

Gráfico 5 - Teste da Razão de Varância para o Ln do preço da

Celulose $\quad 80$

Gráfico 6 - Gráfico de Dispersão e Linha de Tendência Tendência 82

Gráfico 7 - Valoração do Lucro Estocástico - VPL pela TOR

(Opção 1) 90

Gráfico 8 - Valoração do Lucro Estocástico - VPL pela TOR (Opção 2) 91

Gráfico 9 - Região das Curvas de Gatilho para os preços 94

Gráfico 10 - Região das Curvas de Gatilho para os lucros 95 


\section{Símbolos, acrônicos e abreviaturas}

ABRAF - Associação Brasileira de Produtores de Florestas Plantadas

ABTCP - Associação Brasileira Técnica de Celulose e Papel

ADF - Dickey-Fuller Aumentado

BEKP - Bleached Eucalyptus Kraft Pulp

BHKP - Beached Hardwood Kraft Pulp

BNDES - Banco Nacional do Desenvolvimento Econômico e Social BRACELPA - Associação Brasileira de Celulose e Papel

CETESB - Companhia de Tecnologia de Saneamento Ambiental DF - Dickey-Fuller

DJSI - World - Índice Dow Jones de Sustentabilidade Global

ECF - Elemental Chlorine Free

FCD - Fluxo de Caixa Descontado

FGV - Fundação Getulio Vargas

IBRE - Instituto Brasileiro de Economia

IGP-DI - Índice Geral de Preços - Disponibilidade Interna

INPI - Instituto Nacional da Propriedade Industrial

ISE - Índice de Sustentabilidade Empresarial

MAB - Movimento Aritmético Browniano

MGB - Movimento Geométrico Browniano

MMQ - Método dos Mínimos Quadrados

MRM - Movimento de Reversão à Média

NYSE - New York Stock Exchange

SGA - Sistemas de Gestão Ambiental

SMC - Simulação de Monte Carlo

TCF - Totally Chlorine Free

TIR - Taxa Interna de Retorno

TJLP - Taxa de Juros de Longo Prazo

TOR - Teoria das Opções Reais

VPL - Valor Presente Líquido 